\title{
Immune checkpoint inhibitors in melanoma
}

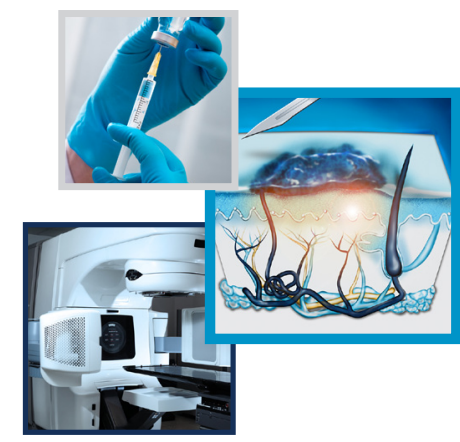

\author{
Adam J Cooper ${ }^{1,2,3}$, Matteo S Carlino ${ }^{* 1,2,3,4}$ \& Richard F Kefford ${ }^{1,2,3,4,5}$
}

\section{Practice points}

- Spontaneous regression of melanoma has been well documented and is most likely immune-mediated.

- IFN- $\alpha$ has modest activity in metastatic melanoma and is used with variable uptake in the adjuvant setting.

- IL-2 can produce durable remissions in a minority of selected patients but toxicity has limited its use.

- Adoptive immunotherapy using autologous tumor-infiltrating lymphocytes can result in complete and durable responses in a minority of selected patients, but is a complex and specialized treatment.

- Melanoma vaccines have shown promise in early trials but those studied in randomized Phase III trials in the adjuvant setting have largely been disappointing.

The potential to harness the power of the immune system and effectively treat patients with metastatic melanoma is finally being realized with the advent of immune checkpoint inhibitors. These new therapies herald a new era in the treatment of melanoma with the potential to produce very durable responses and possible cure for a subset of patients, though bring with them challenges including novel toxicities and nonconventional response patterns. This article reviews the currently available immune checkpoint inhibitors, potential biomarkers to predict response and promising investigational approaches including combination therapies.

Despite decades of attempts it is only recently that real advances have been made in harnessing the power of the immune system to treat metastatic melanoma. Melanoma is notoriously resistant to chemotherapy making immunotherapy an attractive therapeutic approach [1]. Previous strategies have included the use of cytokines, vaccines and adoptive immunotherapy. These therapies have not entered the mainstream either because they failed to demonstrate convincing efficacy or involve complex and toxic regimens.

Immune checkpoints are part of the important mechanism by which self-tolerance is maintained and autoimmune damage of normal tissues is prevented. Tumor cells can hijack this mechanism to develop resistance to immune attack, particularly from $T$ cells. Immune checkpoints involve the binding of a ligand to a receptor, producing an inhibitory signal in immune effector cells which dampens the immune response. Monoclonal antibodies have been designed to bind to the receptors

\section{KEYWORDS}

- anti-CTLA4 - anti-PD-1

- anti-PD-L1 • checkpoint

inhibitor • immunotherapy

- ipilimumab

- melanoma • nivolumab

- pembrolizumab

\section{Future Medicine ${ }_{\text {part of }}$}


or ligands involved, blocking the inhibitory synapse, and releasing T-cell activation.

The first drug shown in a Phase III trial to improve overall survival in advanced melanoma was ipilimumab, an immune checkpoint inhibitor targeting CTLA-4. Ipilimumab has since become a standard therapy for metastatic melanoma and most patients now receive it during the course of their treatment. More recently, the development of antibodies targeting the PD-1 receptor has demonstrated impressive efficacy and low toxicity, and has been hailed as a major advance in cancer therapy.

In contrast to cytotoxic therapies, nonconventional response patterns can be seen with immune checkpoint inhibitors including delayed responses, and new immune-related response criteria have been proposed. These immunotherapies also bring with them a new set of toxicities with the potential to cause a range of immunerelated side effects. Despite these challenges, immune checkpoint inhibitors herald a new era in the treatment of melanoma with the potential to produce very durable responses and possible cure for a subset of patients.

\section{History of immunotherapy in melanoma}

The immune system has long been known to play a role in the natural history of melanoma. Spontaneous partial or complete regression of primary melanomas is well documented and is thought to occur fairly commonly. Complete regression of advanced metastatic disease is less common but has been described many times, with cases reported in the literature as far back as the late 1800s [2].

In a review of the clinical features of spontaneous regression of malignant melanoma, 33 patients with total regression of primary melanoma ('primary regressors') and 40 (13 of whom were somewhat doubtful) with regression of metastatic disease were identified from the literature and reviewed in detail. These patients appeared to represent a typical age incidence of melanoma but the primary regressors showed an unexpected predominance of male over female patients. A variety of unique clinical features of the histories of the patients were noted, but none appeared to explain the regression with any degree of predictability. Cutaneous metastases constituted the most common site of regression, followed, in order, by lymphatic, pulmonary and hepatic metastases. About $40 \%$ of patients with spontaneous regressions appeared to have 'spontaneous cure,' which implies that the disease had not relapsed either during a long period of follow-up or until death from some other cause [3]. Although other pathophysiological mechanisms have been proposed, including endocrinologic and nutritional factors, the most likely is immune-mediated.

It seems likely that some event triggers an increased response from the immune system. A review of reported cases found that the most common factors associated with spontaneous regression were surgical trauma and infection [4].

\section{- IFN- $\alpha$}

IFN- $\alpha$ is a cytokine and was the first such agent used to treat metastatic melanoma. It did not become a standard therapy for metastatic disease as response rates seen in Phase II trials were infrequent (around 15\%), often transient and generally limited to patients with a small volume of soft tissue metastases [5]. These observations did lead to the use of adjuvant IFN- $\alpha$ for resected melanoma at high risk of relapse. Several trials in this setting have shown a significant improvement in relapse-free survival compared with observation, but conflicting results in terms of overall survival [6]. A meta-analysis of mature data from 14 randomized trials involving 8122 patients was able to demonstrate a statistically significant improvement in overall survival as well as relapse-free survival [7]. Of the individual trials, four of 14 found a statistically significant overall survival advantage, and the meta-analysis revealed a significant reduction in the risk of death for patients receiving IFN- $\alpha$ (hazard ratio [HR] for death: $0.89 ; 95 \% \mathrm{CI}$ : $0.83-0.96 ; \mathrm{p}=0.002)$. The likely modest survival benefit and toxicity of IFN- $\alpha$ has led to variable uptake as a standard therapy in the adjuvant setting.

\section{- IL-2}

IL-2 is a cytokine and T-cell growth factor. Early studies showing benefit in a subset of patients with advanced melanoma began almost 30 years ago. A review describing extended follow-up of 270 patients treated with high-dose bolus IL-2 reported that $6 \%$ of patients had complete responses, and a further $10 \%$ had partial responses [8]. The median duration of complete responses had not been reached at last reporting, but exceeded 59 months, and disease progression was not observed in any patient responding for longer than 30 months. IL-2 is a potent inducer 
of other cytokines which can lead to significant toxicity including hypotension, fever, arrhythmias, pulmonary edema and occasionally death. Although high-dose IL-2 has the potential to produce durable remissions in a minority of selected patients, toxicity has limited its use to carefully selected fit patients in specialized centers. Of note there is no randomized clinical study comparing IL-2 to a relevant control arm.

\section{- Adoptive immunotherapy}

Adoptive immunotherapy involves the transfer of harvested immune-derived cells, most commonly $\mathrm{T}$ cells, back into the same patient, or into a new host in the context of a bone marrow transplant. The use of autologous cells is generally preferred to minimize complications from graft-versus-host disease. Early attempts in advanced cancer sought to improve the activity of IL-2 by combining it with IL-2 activated peripheral blood lymphocytes (lymphokineactivated killer or LAK cells). However, clinical trials failed to demonstrate sufficient benefit from the combination of IL-2 and LAK cells over IL-2 alone to justify general clinical use [9].

Subsequent studies have combined IL-2 with autologous tumor-infiltrating lymphocytes (TILs) derived from patients' own tumors and expanded in culture. The overall response rate seen in 86 selected consecutive patients with metastatic melanoma treated with this combination was $34 \%$, with five patients (6\%) achieving a complete response [10]. Improved responses were seen in three subsequent sequential studies which used conditioning immunosuppressive chemotherapy with or without total body irradiation to deplete lymphocytes before the infusion of TILs [11]. The pooled results revealed that 20 of the 93 patients (22\%) achieved a complete response, with 19 having ongoing complete regressions beyond 3 years. These results, in highly selected patients, are certainly promising but need to be confirmed by other centers which will be difficult due to the specialized and complex regimens involved. A Phase III trial is currently underway in Europe comparing adoptive transfer of $\mathrm{T}$ cells to standard ipilimumab therapy (NCT02278887).

Methods have been developed to generate $\mathrm{T}$ cells with increased tumor specificity by genetically modifying receptors on patient lymphocytes, which can then be expanded in vitro and used in adoptive cell transfer protocols [12]. The modifications can be made to the T-cell receptor (TCR) or to create a chimeric antigen receptor (an extracellular domain derived from tumor-specific antibody, linked to an intracellular signaling domain). One study screened human lymphocytes and also immunized transgenic mice to generate TCRs with high avidity for melanoma-associated antigens (MART-1 and gp100). Genes encoding these TCRs were engineered into retroviral vectors and used to transduce autologous peripheral lymphocytes. Objective tumor regressions were seen in 30 and $19 \%$ of patients receiving either the human or mouse TCR, respectively. Toxicity was observed in normal tissues including the skin, ear and eye which sometimes required treatment with local steroid therapy [13].

\section{- Melanoma vaccines}

A number of melanoma-specific vaccines have been studied in patients with metastatic disease or in the adjuvant setting with variable success. A vaccine derived from the melanoma antigen glycoprotein 100 (gp100) was studied in combination with IL-2 in a Phase III trial [14]. Patients with metastatic melanoma were randomized to receive IL-2 alone or IL-2 plus the vaccine. The response rate was $16 \%$ for the combination arm compared with $6 \%$ for IL-2 monotherapy. The response rate was lower than expected for the IL-2 alone arm (which may be due to a modified dosing schedule) but the trial did demonstrate improved efficacy with the addition of the vaccine.

MAGE-3 is expressed on around $70 \%$ of melanoma cells. A recombinant MAGE-3 protein vaccine combined with an adjuvant has shown activity in patients with metastatic melanoma and the ability to elicit MAGE-3-specific antibody and a T-cell response in patients [15]. In a Phase II EORTC study, a gene expression profile was found that was associated with an improved clinical response to the MAGE-3 vaccine. The presence of this gene signature was associated with a significantly prolonged time to treatment failure (10.3 months in those with the characteristic gene signature compared with 2.3 months in those without the signature) $[16,17]$. The results of a randomized Phase III trial of the vaccine in resected stage III melanoma are pending, though it has been announced that the first co-primary endpoint was not met [18].

A polyvalent melanoma cell vaccine consisting of three human melanoma cell lines chosen for their high expression of protein and 
ganglioside antigens was examined in a Phase II trial. This trial found that the median survival of stage IV patients was prolonged to 23.1 months, compared with 7.5 months in a control group of patients previously treated with predominately chemotherapy or intratumoral bacillus Calmette-Guérin (BCG) therapy [19]. A subsequent Phase III trial of the whole-cell vaccine compared with placebo in resected stage III melanoma failed to show a benefit [20].

Gangliosides are glycolipids that are overexpressed on the surface of melanoma cells. The presence of antibody to GM2 ganglioside has been associated with prolonged survival in patients with melanoma, and administering a GM2 containing vaccine is effective in inducing an antibody response to GM2 [21]. However, when the GM2-KLH vaccine was studied in two Phase III trials in the adjuvant setting, it failed to show a survival benefit $[22,23]$.

Phase III trials of vaccines in melanoma have focused on the adjuvant setting and it is not clear why the positive results seen in Phase II trials (often in patients with metastatic disease) did not translate into a clinical benefit in these trials. The Phase III trial of the peptide vaccine gp100 in combination with IL-2 did show a benefit with the addition of the vaccine in these patients with metastatic disease. It may be that vaccination alone is not sufficient and that an effective immunomodulator is required to enhance the immune response.

\section{Antibodies to CTLA-4}

\section{- Mechanism of action}

Cancer cells produce proteins that are antigenic due to mutation, abnormally high expression, production by oncogenic viruses or expression of proteins which are usually limited to embryological development or the testis. Such proteins can be recognized as nonself and activate the immune system. Activation of cellular immunity requires that these proteins are presented to T cells, through a process of displaying peptide fragments on the surface of antigen presenting cells, in the context of the MHC. These interactions are thought to occur in lymph nodes during the immune system's priming Phase. Activation of the $\mathrm{T}$ cell also requires a costimulatory signal which occurs when CD28 on its surface binds to $\mathrm{B} 7$ on the dendritic cell [24]. CTLA-4 is also expressed on T cells and competes with CD28/B7 binding, thereby acting as an inhibitory signal or 'brake' on T-cell activation (Figure 1). Ipilimumab is a monoclonal antibody to CTLA-4 that blocks the inhibitory signal and releases T-cell activation pathways, allowing an immune response to occur against the Tumor cells [25]. However, this can also lead to a loss of self-tolerance and the development of significant immune-related side effects [26].

\section{- Ipilimumab}

The effectiveness of ipilimumab in unresectable stage III or stage IV melanoma was initially demonstrated in two randomized Phase III trials. In a study of patients previously treated for metastatic disease, 676 subjects were randomized, in a 3:1:1 ratio, to receive ipilimumab plus a glycoprotein 100 vaccine (gp100), ipilimumab alone or gp100 alone [27]. Ipilimumab was given every 3 weeks for four cycles at a dose of $3 \mathrm{mg} / \mathrm{kg}$ of bodyweight. The median overall survival was 10.0 months in the ipilimumab plus gp100 group, 10.1 months in the ipilimumab alone group and 6.4 months in the gp100 alone group. The best overall response rate of $10.9 \%$ was seen in the ipilimumab alone group, compared with $1.5 \%$ in the gp100 alone group. Grade 3 or 4 immune-related adverse events occurred in up to $15 \%$ of patients treated with ipilimumab and in 3\% treated with gp100 alone. (Grade 3 or 4 adverse events are severe to life-threatening or disabling, and often require intervention.) There were 14 deaths related to the study drugs $(2.1 \%)$, of which seven were attributed to immunerelated adverse events. The results of this trial led to the approval of ipilimumab in the USA in 2011, and subsequently other jurisdictions including Europe and Australia.

In a second Phase III trial, 502 previously untreated patients with metastatic melanoma were randomly assigned to receive dacarbazine with either ipilimumab $(10 \mathrm{mg} / \mathrm{kg})$ or placebo [28]. Ipilimumab or placebo was given every three weeks for four cycles, and patients who had an objective response or stable disease received maintenance therapy every 12 weeks thereafter. Overall survival was significantly longer in the group receiving ipilimumab plus dacarbazine than in the group receiving dacarbazine alone (11.2 vs 9.1 months; HR for death, $0.72 ; \mathrm{p}<0.001)$. The survival rates in the ipilimumab-dacarbazine group were higher at 1 year ( 47 vs 36\%), 2 years (29 vs 18\%) and 3 years (21 vs $12 \%$ ).

Grade 3 or 4 adverse events occurred more frequently in the ipilimumab-dacarbazine arm 


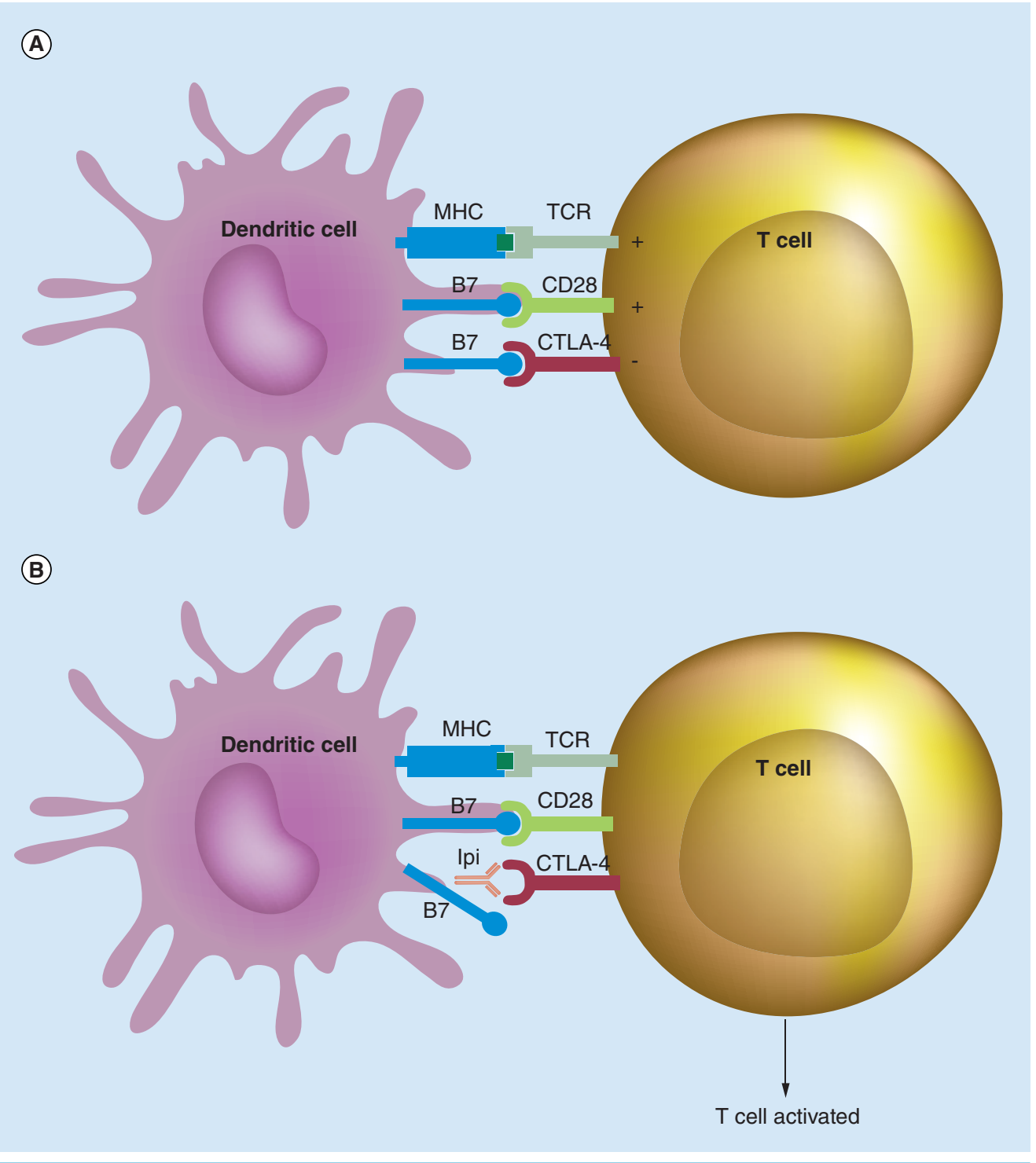

Figure 1. Mechanism of action of ipilimumab. (A) A peptide fragment bound to MHC on the surface of an antigen presenting cell is presented to the T cell. Activation of the T cell requires a co-stimulatory signal which occurs when CD28 binds to B7 on the dendritic cell. CTLA-4 is also expressed on T cells and competes with CD28/B7 binding, acting as an inhibitory signal on T-cell activation. (B) Ipi binds to CTLA-4 and removes the inhibitory signal, activating the $T$ cell and allowing it to proliferate.

Ipi: Ipilimumab.

(56\% of patients) compared with the dacarbazine alone arm (28\% of patients). The incidence of hepatotoxicity seen with ipilimumab in combination with dacarbazine was increased compared with that seen in trials of ipilimumab alone. Due to the increased toxicity and since efficacy does not appear to be significantly improved over ipilimumab alone, the combination of dacarbazine and ipilimumab has not become a standard therapy.
Longer follow-up of patients on this trial revealed that the survival rate for patients treated with ipilimumab appeared to plateau beginning at 3 years [29]. The survival rate of $21.3 \%$ at 3 years in the ipilimumab arm was maintained at $19.1 \%$ at 4 years and $18.2 \%$ at 5 years. A plateau in survival was also observed when long term survival rates in other studies were analyzed. Pooled survival data from 1861 patients treated on 12 prospective and retrospective 
ipilimumab studies revealed a 3-year overall survival rate of $22 \%$ for the entire population, which was maintained at approximately $20 \%$ out to 10 years [30]. The plateau in survival was independent of line of therapy, ipilimumab dose or use of maintenance therapy, and compares favorably to historic long term survival rates of less than $10 \%[31]$.

\section{- Tremelimumab}

Tremelimumab is a human IgG2 monoclonal antibody that also binds and inhibits CTLA-4. Early Phase trials demonstrated activity with objective responses in up to $10 \%$ of patients that were durable (at least 170 days) [32,33]. In contrast to ipilimumab, tremelimumab failed to demonstrate an overall survival benefit in a subsequent Phase III trial [34]. This trial compared tremelimumab, at a dose of $15 \mathrm{mg} / \mathrm{kg}$ every 3 months, to investigator's choice chemotherapy. The median duration of response was 35.8 months for tremelimumab compared with 13.7 months for chemotherapy $(p=0.0011)$, but there was no significant difference in overall survival. The reason for the lack of an overall survival benefit may have been due in part to some patients in the chemotherapy arm crossing over to receive ipilimumab, or potentially to a suboptimal dosing schedule of tremelimumab in the trial [35].

\section{- Dynamics of response}

The effects of cytotoxic treatments are often measurable within a few weeks of starting treatment but it has become clear that responses to immunotherapeutic agents such as ipilimumab can take longer to occur. CTLA-4 inhibitors promote T-cell activation by blocking the inhibitory signal between antigen presenting cells and $\mathrm{T}$ cells that occurs in lymph nodes. It may take some time before sufficient numbers of activated $\mathrm{T}$ cells are created and able to travel to tumor sites throughout the body.

Response patterns seen with ipilimumab differ to those seen with cytotoxic therapy since during the time it takes for the immune system to become activated, lesions may increase in size or new lesions may appear. In the Phase II trials of ipilimumab, unconventional response patterns were observed including: durable stable disease (with a subsequent gradual decline in tumor burden in some patients), a response after an initial increase in tumor burden and response in the presence of new lesions [36,37].
It is important to assess patients being treated with immunotherapies carefully and not to abandon or switch treatment too early, though it should be kept in mind that these patterns of delayed response are unlikely to occur in patients with rapidly progressive, symptomatic disease. Immune-related response criteria have been proposed to deal with the altered patterns of response that can be seen with ipilimumab and potentially other immunotherapies [38]. The criteria are based on WHO response, but differ in that the first assessment is deferred to week 12 , new lesions are considered as part of the total tumor burden and do not automatically indicate progressive disease, and progressive disease is confirmed by repeat imaging at least 4 weeks later.

\section{- Predictors of response}

The Phase III trial of ipilimumab in previously treated patients permitted enrollment of patients with a life expectancy of at least 4 months, good performance status and normal hematological, renal and hepatic function. Subgroup analyses performed in this trial did not reveal any evidence that the survival benefit was restricted to any group based on sex, age ( $\leq 65$ or $>65$ years), stage at presentation (M0, M1a, M1b vs M1c), baseline LDH (normal vs elevated) or prior use of IL-2. Given the patterns of response seen with ipilimumab, however, it is unlikely that patients with rapidly progressive, symptomatic disease will have sufficient time to respond to treatment. Data collected from 153 patients treated on an expanded access program in Spain revealed a median overall survival of 6.5 months and a 1 -year survival rate of $33 \%$, and data from 833 patients on the Italian access program revealed a similar median overall survival of 7.2 months $[39,40]$. Interestingly, an analysis of patients over 70 years of age treated on the Italian program revealed a median overall survival of 8.9 months, indicating ipilimumab is a feasible treatment option in elderly patients [41]. These results are worse than those seen in the Phase III trials of ipilimumab and could be explained by the poorer prognosis and performance status of patients treated on the access programs.

Characteristics of the tumor may predict response to CTLA-4 inhibitors, with a high mutational burden producing a wider range of antigens and an increased immune response. Whole-exome sequencing of DNA from tumors 
of ipilimumab-treated patients has revealed that tumors with higher mutational loads are associated with increased benefit from treatment [42]. However, some tumors with a high mutational burden did not respond to treatment. Further analysis identified peptides predicted to bind to MHC class I molecules present in tumors of patients with a long-term clinical benefit but completely absent in patients with a minimal benefit or no benefit. These neoantigens were used to construct a neoepitope signature that was tested in a validation cohort and correlated very strongly with survival [42]. This research provides proof of principle that tumor genomics can predict response to immunotherapy.

Host factors may also play a role and an interesting observation is that patients who develop immune-related toxicities on treatment may be more likely to respond. In an analysis performed across three Phase II ipilimumab trials, the disease control rate was higher in patients who experienced grade 2-4 immune-related toxicities compared with grade $0-1$ toxicities (34-43\% vs $20-24 \%$ ), but the association was not statistically significant [43]. Thus a correlation between toxicity and clinical efficacy has not been proven, and patients who do not experience an immune-related toxicity may still benefit from ipilimumab.

In a preliminary study, blood samples from 45 patients (21 responders, 24 nonresponders), were analyzed with exome sequencing. Single nucleotide polymorphisms in the chemokine receptors CCR2, CCRL 2 and CCR 5 were associated with nonresponse to anti-CTLA-4 therapy [44]. All these genes are found within a chemokine receptor locus on the short arm of chromosome 3 and are highly correlated. Further validation and functional analysis is required to determine functional significance of these variants. This research does suggest that an inherited mutation in a proinflammatory chemokine receptor may give rise to resistance to CTLA-4 therapy.

There is currently no validated biomarker predictive of response to ipilimumab, though potential candidates have been identified based on preliminary evidence. In a retrospective analysis of immunological parameters in patients treated with ipilimumab on an access program, an increase in circulating inducible T-cell co-stimulator (ICOS)-positive T-cell levels in patients at week 7 was associated with longer survival. A neutrophil:lymphocyte ratio below the median at weeks 7 and 10 was also associated with better survival compared with an elevated ratio [45].

NY-ESO-1 is a cancer/testis antigen expressed in a subset of patients with melanoma. In one study, pre-existing patient immunity to NY-ESO-1 was associated with increased clinical benefit from ipilimumab at 24 weeks, compared with NY-ESO-1-negative patients [46]. Based on this observation, a trial of a vaccine based on the NY-ESO-1 antigen in combination with ipilimumab was designed and is recruiting patients with metastatic melanoma whose tumors express NY-ESO-1 or the related LAGE-1 antigen.

Pretreatment serum VEGF levels were found to be associated with response to ipilimumab in one study [47]. High pretreatment VEGF levels were associated with decreased survival compared with low VEGF levels (median overall survival 6.6 vs 12.9 months; $\mathrm{p}=0.006$ ). This research suggests that there is a potential interaction between the biology of angiogenesis and immune-checkpoint blockade, and warrants prospective investigation.

Levels of circulating myeloid-derived suppressor cells (MDSCs) have been found to be higher in patients with advanced melanoma than healthy donors. Research has found that clinical responders to ipilimumab therapy showed significantly fewer lin(-) $\mathrm{CD}_{14}{ }^{+}$HLA-DR MDSCs as compared with nonresponders [48]. These data suggest that the frequency of circulating MDSCs may prove to be a predictive marker of response, as low levels could identify patients more likely to benefit from ipilimumab treatment. Prospective clinical trials examining MDSC levels as a potential biomarker are needed to validate these observations.

\section{- Toxicities of CTLA-4 inhibitors}

CTLA-4 inhibitors can cause a range of immune-related adverse events including colitis, dermatitis, hepatitis and endocrinopathies such as hypophysitis, thyroiditis and adrenal insufficiency. In the Phase III trials of ipilimumab, grade 3 or 4 immune-related adverse events occurred in 10 to $15 \%$ of patients in the ipilimumab groups [27,28]. High-grade hepatotoxicity was particularly seen in the combined ipilimumab + dacarbazine arm. Immune-related adverse events most often affected the skin and gastrointestinal tract with diarrhea being the most common immune-related toxicity.

It is important to monitor patients closely for immune-related toxicities, particularly colitis, 
as if not treated early colitis can become severe and result in bowel perforation and death. Management algorithms are available for the various immune-related adverse events including diarrhea/colitis [49]. They recommend the use of systemic corticosteroids for severe diarrhea or colitis, and for mild or moderate symptoms that persist or recur. It is important to taper steroid therapy slowly, over at least a month, as colitis can recur if steroids are reduced too quickly. Steroid refractory colitis can be successfully treated with infliximab [50,51]. Severe life-threatening steroidresistant hepatitis or colitis may also be rapidly reversed with antithymocyte globulin [52].

The symptoms of cortisol deficiency due to hypophysitis or adrenal insufficiency can be vague and include fatigue, light-headedness and headaches. A low threshold for investigating these symptoms is needed, and initial investigations should include a cortisol level (preferably in the morning) and thyroid function tests. In patients who develop cortisol deficiency, an adrenocorticotropic hormone (ACTH) level and short ACTH stimulation test can help determine whether the deficiency is due to pituitary (more common) or adrenal failure.

Toxicities such as colitis, dermatitis and hepatitis are generally reversible with steroid treatment, but patients who develop hypophysitis or hypothyroidism usually require life-long hormone replacement therapy. The management of immune-related toxicities can be complex and multi-disciplinary care involving specialists from other relevant fields is ideal.

\section{- Sequencing treatment with BRAF inhibitors}

The optimal sequencing of CTLA- 4 inhibitors and BRAF inhibitors in patients with $B R A F$ mutant melanoma has not been examined prospectively in trials. When the results of the pivotal trials of ipilimumab and the BRAF inhibitor vemurafenib were reported, it was thought that combining both drugs together might be the best strategy for patients with $B R A F$-mutant disease. However, a trial combining these two drugs was stopped early due to unacceptable rates of hepatotoxicity [53]. Preliminary data regarding the combination of ipilimumab and the BRAF inhibitor dabrafenib, with or without the MEK inhibitor trametinib, have been presented. In eight patients treated with dabrafenib in combination with ipilimumab toxicities appeared consistent with single agent data, and further enrollment is ongoing. The triple combination of dabrafenib, trametinib and ipilimumab was associated with colitis and bowel perforation in two of seven patients treated, and no further patients are being enrolled on the triplet combination due to safety concerns [54].

Evidence from retrospective analyses suggests that the sequence of ipilimumab first and then BRAF inhibitor may be superior to the reverse sequence [55,56]. Outcomes for ipilimumab following BRAF discontinuation were poor in two published retrospective analyses, with 43 and $50 \%$ of patients progressing rapidly and being unable to complete four cycles of ipilimumab. A third retrospective study from a single institution reports an overall response rate of $27 \%$ with ipilimumab treatment after BRAF inhibitor failure [57]. Most of the patients included in this study were treated with ipilimumab in combination with a dendritic cell vaccine. The discrepancy between such studies is likely due to selection bias and illustrates the need for a randomized trial to examine the sequencing of targeted therapy and immunotherapy.

The strategy of pre-emptive switch from induction vemurafenib to ipilimumab has been examined. The rationale for this strategy comes from translational studies which show that BRAF inhibitor treatment leads to a marked T-cell tumor infiltrate and increased tumor antigen expression [58,59]. In a single institution study of 19 patients, 11 were pre-emptively switched from vemurafenib to ipilimumab (at 2-4 months); there were no melanoma specific deaths (at a median follow-up of 1 year) in these 11 patients. In the five of 11 who progressed after ipilimumab, retreatment with vemurafenib was possible. Seven of the eight patients switched at progression had died at the time of reporting (median overall survival: 6.6 months) [60]. Selection bias is inherent in these data; however, this strategy warrants further testing within a formalized clinical trial protocol.

Particular challenges are present in managing patients with slow progression on BRAF inhibitors who may be benefiting from BRAF inhibition beyond disease progression [61]. Cessation of BRAF inhibitors in these patients has been associated with well-documented examples of rapid disease progression, which, in certain cases, may restrict the opportunity for immunostimulant therapy [62].

Since response rates to BRAF inhibitors (particularly in combination with a MEK inhibitor) 
are very high but can be short-lived, and response rates to ipilimumab are relatively low but can be very durable, a logical strategy would be to treat with immunotherapy first hoping for a durable response and saving targeted therapy for later use if needed. An exception might be a patient with bulky, symptomatic disease where a rapid response is needed to control the disease, in which case upfront targeted therapy may be preferable. Clinical trials testing the sequencing of immunotherapy and MAPK inhibitors are pending.

\section{Antibodies to PD-1 \& PDL-1}

- Mechanism of action

PD-1 is an immune-inhibitory receptor of the CD28 family expressed by activated $\mathrm{T}$ and $\mathrm{B}$ cells. It has two ligands, PD-L1 and PD-L2, which are members of the B7 family. These ligands are expressed on immune cells such as macrophages and monocytes. Nonimmune cells can also express ligands for PD-1, primarily PD-L1, and this mechanism can be exploited by tumor cells to avoid immune attack [63]. Monoclonal antibodies targeting both PD-1 and PD-L1 have been developed to interrupt this immune-inhibitory pathway and activate the T-cell response against tumor cells. In contrast to CTLA-4 inhibitors which act during the T-cell priming Phase in draining lymph nodes, PD-1/PD-L1 inhibitors act in the effector Phase by blocking T-cell-tumor inhibitory interaction at the tumor level (Figure 2). These antibodies have demonstrated significant clinical activity against multiple tumor types. Melanoma was the outstanding test case for these drugs because it commonly over-expresses PD-L1 and because there were few other active systemic drug treatments for it, apart from those targeting the 50\% of tumors with mutant BRAF.

\section{- Anti-PD-1 antibodies \\ Pembrolizumab}

Pembrolizumab (MK-3475, temporarily known as lambrolizumab) has been evaluated in both ipilimumab naive and previously treated patients [64]. An updated analysis of 411 patients with advanced melanoma treated on this study was presented at the 2014 American Society of Clinical Oncology (ASCO) meeting [65]. Among the 365 patients with measurable disease at baseline, the objective response rate was $40 \%$ in ipilimumab naive patients, and $28 \%$ in ipilimumab pretreated patients. Responses were durable with
$88 \%$ ongoing at analysis. Median overall survival was not yet reached, and the 1-year survival rate was $71 \%$. Drug-related grade 3 or 4 adverse events were experienced in $12 \%$ of patients.

There were no significant differences seen in outcomes between the three different dosing schedules used $(10 \mathrm{mg} / \mathrm{kg}$ every 2 weeks, $10 \mathrm{mg} / \mathrm{kg}$ every 3 weeks or $2 \mathrm{mg} / \mathrm{kg}$ every 3 weeks). In a published analysis of 173 patients from this trial with ipilimumab resistant disease who were randomly assigned to either 2 or $10 \mathrm{mg} / \mathrm{kg}$ given every 2 weeks, there was no difference in the objective response rate between the two schedules [66].

In a randomized Phase II study, patients who had confirmed progression within 6 months of treatment with ipilimumab were randomized to either pembrolizumab (2 mg/kg or $10 \mathrm{mg} / \mathrm{kg}$ every 3 weeks) or physician's choice chemotherapy. Pembrolizumab was associated with an improved response rate $(21,25$ and $4 \%$, for pembrolizumab $2 \mathrm{mg} / \mathrm{kg}$, pembrolizumab $10 \mathrm{mg} / \mathrm{kg}$, and chemotherapy, respectively). 6-month progression free survival rates were also improved at 34,38 and $16 \%$ (for $2 \mathrm{mg} / \mathrm{kg}, 10 \mathrm{mg} / \mathrm{kg}$, and chemotherapy, respectively) [67].

The randomized Phase III study of ipilimumab versus pembrolizumab has completed accrual, with results awaited (NCT01866319). Pembrolizumab was approved by the US FDA for treatment of patients with advanced or unresectable melanoma after failure of prior therapy in September 2014.

\section{Nivolumab}

The efficacy of nivolumab has been demonstrated in a Phase I/II dose escalation, cohort expansion study including 107 patients with melanoma [68,69]. Patients received intravenous nivolumab every 2 weeks for up to 96 weeks. The updated objective response rate seen in melanoma patients was $31 \%$. The median overall survival was 16.8 months, and the 1 - and 2 -year survival rates were 62 and $43 \%$, respectively. Responses frequently persisted after treatment discontinuation, with 12 of 17 patients who stopped treatment for reasons other than progression continuing to respond for at least 16 weeks. The most common adverse events were fatigue, rash, and diarrhea, and serious (grade 3 or 4) treatment-related adverse events occurred in $22 \%$ of patients. The response and survival rates seen with nivolumab in this trial were impressive, especially when taking into 


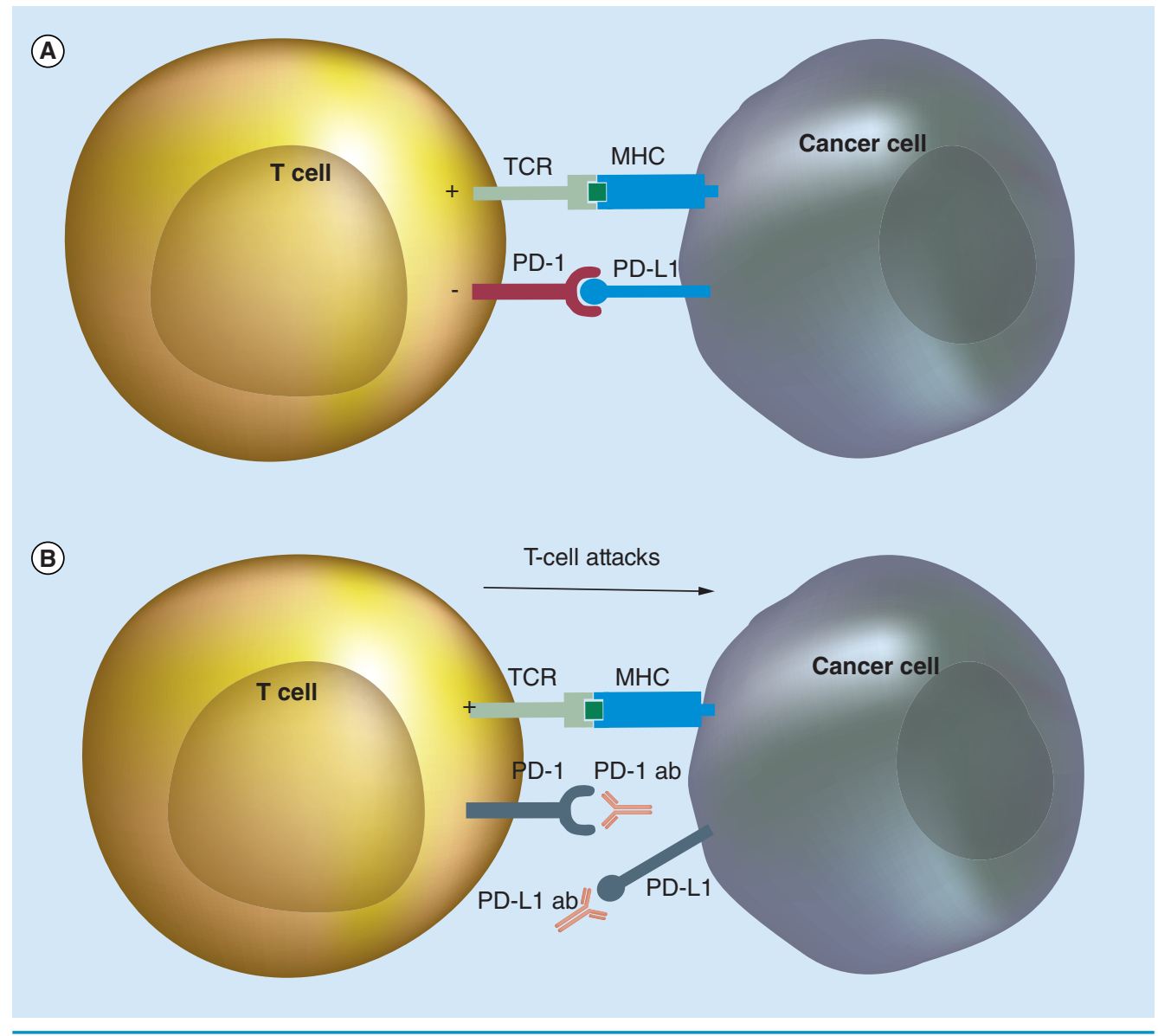

Figure 2. Mechanism of action of antibodies to PD-1 and PDL-1. (A) The T cell recognizes a cancer cell expressing an antigen which has been presented to it previously. The cancer cell expresses PD-L1 which binds to PD-1 and acts as an inhibitory signal. (B) The anti-PD-1 antibody binds to PD-1 (or anti-PD-L1 binds to PD-L1) and removes the inhibitory signal, allowing the T cell to attack the cancer cell.

consideration that the majority of patients were heavily pretreated (62\% with two to five prior systemic therapies).

A Phase III trial has examined the efficacy of nivolumab compared with chemotherapy after failure of prior ipilimumab therapy (and a BRAF inhibitor if $B R A F$-mutant) [70]. Patients were randomized in a $2: 1$ ratio to receive either nivolumab ( $3 \mathrm{mg} / \mathrm{kg}$ every 2 weeks) or investigator's choice chemotherapy (dacarbazine or carboplatin plus paclitaxel). The objective response rate was 32\% in the nivolumab group, compared with $11 \%$ in the chemotherapy group. Among patients followed up for at least 6 months, the median duration of response was 3.6 months in the chemotherapy group and not reached in the nivolumab group (with $95 \%$ of patients still responding). Overall survival data were pending at the time of last reporting.
The efficacy of nivolumab in the first-line setting has been demonstrated in the Phase III CheckMate 066 trial [71]. A total of 418 previously untreated patients with $B R A F$ wild-type metastatic melanoma were randomized to either nivolumab ( $3 \mathrm{mg} / \mathrm{kg}$ every 2 weeks) or dacarbazine chemotherapy. The objective response rate was $40 \%$ in the nivolumab group compared with $14 \%$ in the dacarbazine group (odds ratio: 4.06; $\mathrm{p}<0.001)$. The 1 -year overall survival rate was $73 \%$ in the nivolumab group, compared with $42 \%$ in the dacarbazine group (HR for death: 0.42 ; 99.79\% CI: $0.25-0.73$; $<<0.001$. The median overall survival was 10.8 months in the dacarbazine group, and not reached in the nivolumab group (at a median follow-up of 9 months). Grade 3 or 4 drug-related adverse events occurred in $11.7 \%$ of the patients treated with nivolumab and $17.6 \%$ of those treated 
with dacarbazine. The efficacy of nivolumab in combination with ipilimumab is discussed later. Nivolumab received FDA approval for the treatment of patients with advanced or unresectable melanoma after failure of prior therapy in December 2014.

\section{Pidilizumab}

Pidilizumab is a humanized anti-PD-1-antibody which has been previously shown to have activity in lymphoma. A Phase II study has been performed in melanoma patients who were randomized to one of two dosing regimens [72]. 103 patients were randomized, of whom $51 \%$ had received prior ipilimumab. The overall response rate for all patients was $6 \%$ and the overall survival at 12 months was $65 \%$. There were no significant differences between the two dosing levels, or between those who were ipilimumab naive versus ipilimumab treated.

\section{- Anti-PD-L1 antibodies}

The anti-PD-L1 antibody BMS-936559 has been examined in a Phase I trial involving patients with several different advanced cancers [73]. The trial included 55 patients with melanoma. Among those melanoma patients with evaluable disease, objective responses were seen in nine of 52 patients, and five of these had an objective response lasting at least 1 year. Three patients had a complete response. The objective response rate of $17 \%$ (an average over three dose schedules) seen in melanoma patients was higher than in the other cancer types included in the study. Drug-related grade 3 or 4 toxic events occurred in $9 \%$ of patients overall.

The results from a Phase I dose-escalation study of another PD-L1 antibody, MPDL3280A, were presented at the 2013 ASCO meeting [74]. In the 35 metastatic melanoma patients treated at doses of between 1 and $20 \mathrm{mg} / \mathrm{kg}$ who were evaluable for efficacy, an objective response rate of $26 \%$ (9/35) was observed. All responses were ongoing or improving at the time of reporting, and some patients had tumor shrinkage within days of starting treatment. The incidence of all grade 3 or 4 adverse events, regardless of attribution, was $33 \%$. There were no cases of severe pneumonitis or treatment-related deaths.

The activity of these agents in melanoma patients is promising, although the response rates do appear to be lower than those seen with anti-PD-1 antibodies.

\section{- Toxicities}

The side effect profile of PD-1 and PD-L1 inhibitors is quite favorable compared with that of the CTLA-4 inhibitors. This may be due to the fact that PD-1/PD-L1 inhibitors act during the effector Phase at the tumor level resulting in a relatively specific antitumor response, in contrast to CTLA-4 inhibitors which induce T-cell activation in the priming Phase resulting in less specific immune activation. The potential toxicity of most concern for patients receiving ipilimumab is colitis since it has been associated with fatal perforations. PD-1 inhibitors can cause similar immune-related side effects as those seen with ipilimumab, but severe toxicities occur much less frequently. The most concerning potential toxicity of PD-1 inhibitors is pneumonitis as there have been rare fatal cases documented.

In the Phase I trial of nivolumab in patients with several cancer types, the most common toxicities reported were diarrhea, rash and pruritus, and were mostly low grade [69]. Hypothyroidism or hyperthyroidism occurred in 3\% of patients overall. Pneumonitis occurred in 3\% of patients overall and three patients died of pulmonary toxicity. None of the fatal cases were melanoma patients, however, and two were lung cancer patients who it seems may be more prone to develop pneumonitis on treatment. Longer follow-up of the melanoma patient cohort confirmed that there were no drug-related deaths in this group and no cases of high-grade pneumonitis [68].

Patients with low-grade pneumonitis were successfully managed by interruption of treatment, corticosteroid therapy or both. In the Phase I trial of pembrolizumab the most common toxicities were fatigue, rash, pruritus and diarrhea which were mostly low grade [64]. Pneumonitis was seen in $4 \%$ of patients but no severe cases were reported. Autoimmune synovitis has been reported in patients receiving pembrolizumab [75].

Immune-related toxicities that can occur with ipilimumab including colitis, hepatitis, hypophysitis and thyroiditis can occur with PD-1 inhibitors but generally much less frequently than with ipilimumab. For both pembrolizumab and nivolumab the incidence of any individual toxicity at a severe level (grade 3 to 4 ) was approximately $1 \%$ or less. The low toxicity of anti-PD-1 antibodies has made it feasible to trial them in combination with other agents. 
- PD-1 antibodies \& ipilimumab in combination

The combination of anti-CTLA-4 immunotherapy with ipilimumab plus anti-PD-1 immunotherapy with nivolumab appears to be more active than either drug alone but at the expense of increased toxicity [76]. This combination was assessed in a Phase I dose escalation study. In a concurrent cohort of 53 patients, both drugs were given every 3 weeks for 4 cycles, then nivolumab alone every 3 weeks until week 24 and then both drugs every 12 weeks for a maximum of 8 cycles. A sequential cohort of 33 patients who had already received ipilimumab was treated with nivolumab alone every 2 weeks for up to 48 doses.

In the concurrent group, $53 \%$ of patients had an objective response at the maximum doses associated with an acceptable level of adverse events (nivolumab $1 \mathrm{mg} / \mathrm{kg}$ and ipilimumab $3 \mathrm{mg} / \mathrm{kg}$ ). These were all deep responses with tumor reductions of $80 \%$ or more, and three patients had a complete response. In an update presented at the 2014 ASCO meeting, the 1-year overall survival rate was $94 \%$ in this cohort (and $82 \%$ for the concurrent cohort overall). The median overall survival was not yet reached in this group, but was 40 months in the concurrent cohort overall [77]. The objective response rate in the sequential cohort was lower at $20 \%$. Responses were similar between patients with or without $B R A F$-mutated tumors.

Overall in the concurrent cohort, $53 \%$ of patients experienced grade 3 or 4 adverse events related to therapy. The adverse events were similar in quality to those seen with monotherapy and were considered manageable. The rate is higher than that seen in other monotherapy trials, for example, around $15 \%$ of patients experiencing grade 3 to 4 drug-related events with ipilimumab. Among patients in the sequential cohort, 18\% experienced drug-related grade 3 or 4 adverse events.

The efficacy results are clearly impressive with 1-year survival rates at unprecedented levels for patients with metastatic melanoma, however this combination needs to be formally compared with monotherapy in a larger group of patients before becoming a standard treatment. A three armed Phase III trial is currently underway comparing the combination of nivolumab and ipilimumab, to either drug alone (NCT01844505).

\section{- Biomarkers}

To evaluate whether the expression of the PD-L1 ligand on tumor cells correlates with response to PD-1 inhibitors, pretreatment biopsy samples from a subset of treated patients have been examined. In the Phase I nivolumab study, pretreatment biopsies were collected from 42 patients and 25 were found to be positive for PD-L1 expression by immunohistochemical analysis. Of these 25 patients, nine (36\%) had an objective response while none of the patients with PD-L1-negative tumors had an objective response [69]. Given the nonrandom collection of biopsies and relatively small numbers of patients these results were considered hypothesis generating rather than definitive.

Further research on tumor specimens from patients treated on the above trial evaluated other factors in the tumor microenvironment including PD-1, PD-L1 and PD-L2 expression by tumor cells and infiltrating immune cells [78]. PD-1 expression by TILs was significantly associated with PD-L1 expression by tumor cells and by immune cell infiltrates, suggesting presence of an immunosuppressive tumor microenvironment prior to therapy. Expression of the PD-1 receptor on TILs, however, had only a borderline association with clinical response to nivolumab. Expression of PD-L2 (the second ligand for PD-1) on tumor and immune cells was associated with PD-L1 expression, but was observed less frequently. Tumor PD-L1 expression was the single factor most closely correlated with response to nivolumab. Further research is needed in larger cohorts of patients to determine whether a panel of pretreatment tumor markers may have more powerful associations with clinical outcomes.

PD-L1 expression in the tumor has also been examined as a predictive marker for responsiveness to pembrolizumab. Biopsies were required within 60 days of commencing treatment on the Phase I trial, and results have been reported in 71 patients with measurable disease in which PD-L1 expression was evaluable [79]. Of these patients, 77\% were considered PD-L1-positive (based on a cut-off of $1 \%$ of stained tumor cells by immunohistochemical), and these patients had an improved objective response rate (51 vs $6 \%$ ), and higher 1-year overall survival rate (84 vs $69 \%$ ) compared with PD-L1-negative patients. Although tumor PD-L1 positivity was associated with improved response and survival rates, activity was also observed in patients with low baseline PD-L1 expression treated with pembrolizumab.

The PD-1/PD-L1 interaction and the relationship to clinical response were examined 
further in a study of 46 patients treated with pembrolizumab who underwent tumor biopsies before and during treatment [80]. Tissue samples were analyzed for the presence of $\mathrm{CD} 8^{+} \mathrm{T}$ cells (TILs) before and during anti-PD-1 therapy. Pretreatment samples of patients who responded to pembrolizumab showed higher numbers of $\mathrm{CD} 8^{+} \mathrm{T}$ cells at the invasive margin of the tumor when compared with samples from nonresponders. In other words, low TILs were associated with lack of response to anti-PD-1 therapy, and the authors have suggested that combining PD-1 blockade with therapies that can induce a typeI interferon inflammatory response should be further investigated.

The usefulness of PD-L1 as a biomarker remains to be determined. The various methodological approaches used to examine PD-L1 expression have led to uncertainty regarding the usefulness of PD-L1 expression as a biomarker. Differences include the antibody used, cut-offs for positive expression and tissues included in assessment (tumor only or tumor and stroma). Certainly in the first study to examine the biomarker in prespecified subgroups in a randomized Phase III trial, nivolumab was associated with an improved response rate and progression free survival irrespective of PD-L1 status [71]. Further research will be needed to determine whether tumor PD-L1 expression, or other factors including immune infiltrates or PD-1 expression [78], can be used to guide treatment decisions in melanoma patients, since the available data are not sufficient to deny a patient treatment with a PD-1 inhibitor based on negative PD-L1 staining.

\section{Future directions}

\section{- Adjuvant trials}

Given the clear survival benefits seen with checkpoint inhibitors in the metastatic setting it is logical to study whether using these drugs in the adjuvant setting can improve progression-free and overall survival. A Phase III trial is comparing ipilimumab to placebo after complete resection of stage III melanoma, and preliminary efficacy and safety results were presented at the 2014 ASCO meeting [81]. In this trial, 951 patients were randomized to receive ipilimumab $10 \mathrm{mg} / \mathrm{kg}$ or placebo every 3 weeks for 4 doses, then every 3 months for up to 3 years (until completion, disease recurrence or unacceptable toxicity).

At a median follow-up of almost 3 years, the median recurrence-free survival was significantly longer in the ipilimumab arm at 26.1 months compared with 17.1 months in the placebo arm (HR: 0.75; 95\% CI: 0.64-0.90; $\mathrm{p}=0.0013$ ). The benefit was consistent across subgroups (e.g., stage IIIa or IIIb) though the benefit may be more marked in patients with an ulcerated primary. It remains to be seen whether this benefit translates into an overall survival advantage. Toxicity was notable with $52 \%$ of patients in the ipilimumab arm discontinuing treatment due to adverse events, and five patient deaths (1.1\%) due to drug-related toxicity.

The toxicity and cost of adjuvant ipilimumab will mean that the survival benefit (if observed) will have to be weighed against these factors. Another trial is underway comparing two different dosing schedules of ipilimumab $(10 \mathrm{mg} /$ $\mathrm{kg}$ or $3 \mathrm{mg} / \mathrm{kg}$ ) and high-dose IFN- $\alpha$ in completely resected stage III-IV melanoma (NCT 01274338). This trial will help determine whether a less toxic dose of ipilimumab is effective, and whether it is superior to IFN- $\alpha$. Since anti-PD-1 antibodies are better tolerated than ipilimumab they may have a more favorable riskbenefit ratio in the adjuvant setting, and trials involving PD-1 inhibitors are planned.

\section{- Combination therapies}

The combination of PD-1 and CTLA- 4 inhibitors shows great promise in terms of efficacy, though combination therapy will come with the expense of increased toxicity. The randomized Phase III trial comparing nivolumab, ipilimumab, and the combination of both is underway and results are eagerly awaited. A study examining the combination of pembrolizumab and ipilimumab has recently opened (NCT02089685). As mentioned previously, a Phase I trial of the combination of ipilimumab and dabrafenib is in progress, but the arm examining the triplet combination of ipilimumab, dabrafenib and trametinib was closed to recruitment due to safety concerns (NCT01767454) [54]. A study of the combination of pembrolizumab with dabrafenib and/or trametinib is currently accruing participants (NCT02130466).

A recently published Phase II has examined the combination of ipilimumab with sargramostim, a granulocyte-macrophage colonystimulating factor (GM-CSF) [82]. The trial randomized 245 patients to receive either ipilimumab $(10 \mathrm{mg} / \mathrm{kg})$ plus sargramostim $(250 \mathrm{mg}$ subcutaneously on days 1-14) every 3 weeks for four cycles, or ipilimumab alone, followed by 
maintenance ipilimumab every 12 weeks. At a median follow-up of 13 months, median overall survival was significantly improved at 17.5 months with the combination, compared with 12.7 months for ipilimumab alone. However, the objective response rate was the same in both arms at $15 \%$. Toxicity was lower in the combination arm with $45 \%$ of patients experiencing grade 3 or higher adverse events, compared with $58 \%$ in the ipilimumab alone arm. The dose of ipilimumab used in the study is higher than the approved dose and maintenance therapy was also given, meaning further study is needed before this strategy could be incorporated into standard practice. It is also not clear how much of the improvement in overall survival was a result of the reduction in serious adverse events brought about by the addition of GM-CSF.

The combination of talimogene laherparepvec (T-VEC) with ipilimumab has also demonstrated promise. T-VEC is an HSV-1-derived oncolytic immunotherapy that has previously been shown to have activity in metastatic melanoma. In a Phase Ib study of 19 patients, T-VEC was given intralesionally and starting prior to ipilimumab therapy. The objective response rate for the combination was $41 \%$ [83]. Two patients (11\%) had suspected grade 3 or 4 immunerelated adverse events. Flow cytometry was performed on blood samples and revealed that activated $\mathrm{CD}^{+} \mathrm{T}$ cells significantly increased from baseline during T-VEC treatment, and levels further increased during combination therapy. T-VEC is also being explored in combination with pembrolizumab (NCT 02263508).

Radiotherapy can have beneficial immunogenic effects and may improve responses when used in combination with immunotherapy. There are several reported cases and retrospective series suggesting that radiotherapy can enhance the efficacy of immunotherapy in patients with melanoma, and appears to be safe [84-86]. There are no prospective clinical data available as yet, but trials are underway.

\section{- Other immune checkpoint agents}

CD137 is a member of the TNF family and acts as a costimulatory molecule. BMS-663513 is an anti-CD137 monoclonal antibody that stimulates $\mathrm{T}$ cells leading to enhanced IFN- $\gamma$ production. It has been studied in a Phase I dose escalation study in cancer patients in which three partial responses were observed among 54 patients with melanoma [87]. Fatigue, reversible transaminitis and neutropenia were the most common drug-related toxicities. Results from the Phase II expansion in melanoma patients have not yet been published.

CTLA-4 and PD-1 are members of the CD28/CTLA-4 immunoglobulin superfamily and other members of this family may also be important targets for new therapies. Preclinical research has shown that ICOS has an important role in antitumor effect of CTLA-4 blockade. CTLA-4 blockade in combination with tumor cell vaccines engineered to express ICOS ligand enhanced antitumor responses in melanoma and prostate cancer in mice [88]. B- and T-cell attenuator is an inhibitory receptor that when activated inhibits the function of human CD8 + cancerspecific $\mathrm{T}$ cells, and hence is another target for immunotherapy [89].

The immune synapse is highly complex with multiple potential ligand-receptor interactions serving as potential targets for drug interaction [90]. Prioritizing these for drug development remains a major challenge for cancer immunotherapy.

\section{Conclusion}

The potential to harness the power of the immune system and effectively treat patients with melanoma is finally being realized after decades of research into various aspects of the immune response to cancer. Clinical trials of CTLA-4, PD-1 and PD-L1 blocking antibodies have proven the effectiveness of immune checkpoint inhibitors in melanoma and permanently changed the treatment landscape. Most patients now diagnosed with metastatic melanoma will receive treatment with an immune checkpoint inhibitor and many patients will benefit through extended survival and possibly even cure. These antibodies may also prove to have an important role in the adjuvant setting, further improving outcomes in melanoma patients.

Immune checkpoint inhibitors bring with them new toxicities which are very different to those experienced with other treatments and require a new set of skills to manage proficiently. Patterns of response can differ to traditional cytotoxic therapies and this needs to be appreciated when making treatment decisions for patients. The optimal sequence of immunotherapy with BRAF inhibitors is yet to be determined in a prospective trial, and future research will also need to focus on rational combinations to improve outcomes. Since there are some patients who do 
not respond to immunotherapy, further work is needed to identify reliable predictive biomarkers.

\section{Future perspective}

Checkpoint inhibitors have transformed the treatment of metastatic melanoma and provided patients with the potential for very durable responses and possibly cure. Currently the PD-1 antibodies pembrolizumab and nivolumab are approved for use after failure of prior treatment, but as more evidence of their efficacy in the firstline setting emerges, it is likely that they will become part of the standard initial therapy for metastatic melanoma. It is also likely that combination therapies will become more commonly used as we strive to improve response rates and long-term survival. This will need to be tempered with the increased risk of side effects and may limit combination therapy to fit patients. In patients with $B R A F$-mutant disease, the combination or sequencing of a BRAF inhibitor with a PD-1 antibody may prove to transform the high response rates seen with BRAF inhibition into high rates of durable response with immunotherapy. There is a strong need for biomarkers that will predict response to checkpoint inhibitors and the extensive research into novel biomarkers such as gene expression signatures should yield results. Molecular analyses of mechanisms of resistance to ipilimumab and anti-PD1 drugs promise to inform future rational selection from a growing array of additional immunomodulators to take forward into clinical trial.

\section{Financial \& competing interests disclosure} AJ Cooper discloses that he has received honoraria from Bristol-Myers Squibb Australia (for providing education related to ipilimumab therapy to nursing and medical staff). MS Carlino and RF Kefford are consultant advisors for Bristol-Myers Squibb and Merck Pharmaceuticals. The authors have no other relevant affiliations or financial involvement with any organization or entity with a financial interest in or financial conflict with the subject matter or materials discussed in the manuscript apart from those disclosed.

No writing assistance was utilized in the production of this manuscript.

\section{References}

Papers of special note have been highlighted as:

- of interest; $\bullet$ of considerable interest

1 Eggermont AM, Kirkwood JM. Re-evaluating the role of dacarbazine in metastatic melanoma: what have we learned in 30 years? Eur. J. Cancer 40(12), 1825-1836 (2004).

2 Bennet WH. Some peculiarities in the behaviour of certain malignant and innocent growths. Lancet 1, 3-7 (1899).

3 Nathanson. Spontaneous regression of malignant melanoma: a review of the literature on incidence, clinical features, and possible mechanisms. Natl Cancer Inst. Monogr. 44, 67-76 (1976).

4 Kalialis LV, Drzewiecki KT, Klyver H. Spontaneous regression of metastases from melanoma: review of the literature. Melanoma Res. 19(5), 275-282 (2009).

5 Creagan ET, Ahmann DL, Frytak S, Long HJ, Chang MN, Itri LM. Three consecutive Phase II studies of recombinant interferon alfa-2a in advanced malignant melanoma. Updated analyses. Cancer 59(3 Suppl.), 638-646 (1987).

6 Ascierto PA, Gogas HJ, Grob JJ et al. Adjuvant interferon alfa in malignant melanoma: an interdisciplinary and multinational expert review. Crit. Rev. Oncol. Hematol. 85(2), 149-161 (2013).
7 Mocellin S, Pasquali S, Rossi CR, Nitti D. Interferon alpha adjuvant therapy in patients with high-risk melanoma: a systematic review and meta-analysis. J. Natl Cancer Inst. 102(7), 493-501 (2010).

8 Atkins MB, Kunkel L, Sznol M, Rosenberg SA. High-dose recombinant interleukin-2 therapy in patients with metastatic melanoma: long-term survival update. Cancer J. Sci. Am. 6(Suppl. 1), S11-S14 (2000).

9 Rosenberg SA, Lotze MT, Muul LM et al. A progress report on the treatment of 157 patients with advanced cancer using lymphokine-activated killer cells and interleukin-2 or high-dose interleukin-2 alone. N. Engl. J. Med. 316(15), 889-897 (1987).

10 Rosenberg SA, Yannelli JR, Yang JC et al. Treatment of patients with metastatic melanoma with autologous tumor-infiltrating lymphocytes and interleukin 2. J. Natl Cancer Inst. 86(15), 1159-1166 (1994).

11 Rosenberg SA, Yang JC, Sherry RM et al. Durable complete responses in heavily pretreated patients with metastatic melanoma using T-cell transfer immunotherapy. Clin. Cancer Res. 17(13), 4550-4557 (2011).

12 Kershaw MH, Westwood JA, Slaney CY, Darcy PK. Clinical application of genetically modified T cells in cancer therapy. Clin. Transl. Immunol. 3(5), e16 (2014).
13 Johnson LA, Morgan RA, Dudley ME et al. Gene therapy with human and mouse T-cell receptors mediates cancer regression and targets normal tissues expressing cognate antigen. Blood 114(3), 535-546 (2009).

14 Schwartzentruber DJ, Lawson DH, Richards JM et al. gp100 peptide vaccine and interleukin-2 in patients with advanced melanoma. N. Engl. J. Med. 364(22), 2119-2127 (2011).

15 Vantomme V, Dantinne C, Amrani N et al. Immunologic analysis of a Phase I/II study of vaccination with MAGE-3 protein combined with the AS02B adjuvant in patients with MAGE-3-positive tumors. J. Immunother. 27(2), 124-135 (2004).

16 Kruit W, Suciu S, Dreno B et al. Immunization with recombinant MAGE-A3 protein combined with adjuvant systems AS15 or AS02B in patients with unresectable and progressive metastatic cutaneous melanoma: a randomized open-label Phase II study of the EORTC Melanoma Group (16032-18031). Presented at: 44th Annual Meeting of the American Society of Clinical Oncology. Chicago, IL, USA, 30 May-3 June 2008.

17 Louahed J, Gruselle O, Gaulis S et al. Expression of defined genes identified by pretreatment tumor profiling: association 
with clinical responses to the GSK MAGE-A3 immunotherapeutic in metastatic melanoma patients (EORTC 16032-18031). Presented at: 44th Annual Meeting of the American Society of Clinical Oncology. Chicago, IL, USA, 30 May-3 June 2008.

18 GSK - The investigational MAGE-A3 antigen-specific cancer immunotherapeutic does not meet first co-primary endpoint in Phase III melanoma clinical trial. www.gsk.com

19 Morton DL, Hoon DS, Nizze JA et al. Polyvalent melanoma vaccine improves survival of patients with metastatic melanoma. Ann. NY Acad. Sci. 690, 120-134 (1993).

20 Morton Dl MN, Thompson JF, Kelley MC, Faries M, Wagner J. An international randomized, Phase III trial of bacillus Calmette-Guerin (BCG) plus allogeneic melanoma vaccine (MCV) or placebo after complete resection of melanoma metastatic to regional or distant sites. J. Clin. Oncol. 25(18S Suppl.), 8508 (2007).

21 Livingston PO, Wong G, Adluri S et al. Improved survival in stage III melanoma patients with GM2 antibodies: a randomized trial of adjuvant vaccination with GM2 ganglioside. J. Clin. Oncol. 12(5), 1036-1044 (1994).

22 Eggermont AM, Suciu S, Rutkowski P et al. Adjuvant ganglioside GM2-KLH/QS-21 vaccination versus observation after resection of primary tumor $>1.5 \mathrm{~mm}$ in patients with stage II melanoma: results of the EORTC18961 randomized Phase III trial. J. Clin. Oncol. 31(30), 3831-3837 (2013).

23 Kirkwood JM, Ibrahim JG, Sosman JA et al. High-dose interferon alfa-2b significantly prolongs relapse-free and overall survival compared with the GM2-KLH/QS-21 vaccine in patients with resected stage IIB-III melanoma: results of intergroup trial E1694/ S9512/C509801. J. Clin. Oncol. 19(9), 2370-2380 (2001).

24 Nurieva RI, Liu X, Dong C. Yin-Yang of costimulation: crucial controls of immune tolerance and function. Immunol. Rev. 229(1), 88-100 (2009).

25 Melero I, Hervas-Stubbs S, Glennie M, Pardoll DM, Chen L. Immunostimulatory monoclonal antibodies for cancer therapy. Nat. Rev. Cancer 7(2), 95-106 (2007).

26 Weber J. Ipilimumab: controversies in its development, utility and autoimmune adverse events. Cancer Immunol. Immunother. 58(5), 823-830 (2009).

27 Hodi FS, O'day SJ, Mcdermott DF et al. Improved survival with ipilimumab in patients with metastatic melanoma. N. Engl. J. Med. 363(8), 711-723 (2010).

•- This seminal paper reported on a Phase III ipilimumab trial that was the first to show a survival benefit in metastatic melanoma.

28 Robert C, Thomas L, Bondarenko I et al. Ipilimumab plus dacarbazine for previously untreated metastatic melanoma. $N$. Engl. J. Med. 364(26), 2517-2526 (2011).

29 Maio M, Bondarenko I, Robert C et al. Survival analysis with 5 years of follow-up in a Phase III study of ipilimumab and dacarbazine in metastatic melanoma. Presented at: ESMO European Cancer Congress 2013. Amsterdam, Netherlands, 27 September-1 October 2013 (Abstract 3704).

30 Schadendorf D, Hodi FS, Robert C et al. Pooled analysis of long-term survival data from Phase II and Phase III trials of ipilimumab in metastatic or locally advanced, unresectable melanoma. Presented at: ESMO European Cancer Congress 2013. Amsterdam, Netherlands, 27 September-1 October 2013 (Abstract LBA 24).

- This review demonstrates the durable nature of responses to ipilimumab therapy with the 3 year survival rate of around $20 \%$ maintained out to 10 years.

31 Balch CM, Buzaid AC, Soong SJ et al. Final version of the American Joint Committee on Cancer staging system for cutaneous melanoma. J. Clin. Oncol. 19(16), 3635-3648 (2001).

32 Camacho LH, Antonia S, Sosman J et al. Phase I/II trial of tremelimumab in patients with metastatic melanoma. J. Clin. Oncol. 27(7), 1075-1081 (2009).

33 Kirkwood JM, Lorigan P, Hersey $\mathrm{P}$ et al. Phase II trial of tremelimumab (CP-675,206) in patients with advanced refractory or relapsed melanoma. Clin. Cancer Res. 16(3), 1042-1048 (2010).

34 Ribas A, Kefford R, Marshall MA et al. Phase III randomized clinical trial comparing tremelimumab with standard-of-care chemotherapy in patients with advanced melanoma. J. Clin. Oncol. 31(5), 616-622 (2013).

35 Ribas A, Hauschild A, Kefford R, Wilson KS et al. J. Clin. Oncol. 31(22), 2836-2837 (2013).

36 O'day SJ, Maio M, Chiarion-Sileni V et al. Efficacy and safety of ipilimumab monotherapy in patients with pretreated advanced melanoma: a multicenter single-arm Phase II study. Ann. Oncol. 21(8), 1712-1717 (2010).

37 Wolchok JD, Neyns B, Linette G et al. Ipilimumab monotherapy in patients with pretreated advanced melanoma: a randomised, double-blind, multicentre, Phase II, dose-ranging study. Lancet Oncol. 11(2), 155-164 (2010).

38 Wolchok JD, Hoos A, O'day S et al. Guidelines for the evaluation of immune therapy activity in solid tumors: immunerelated response criteria. Clin. Cancer Res. 15(23), 7412-7420 (2009).

39 Ascierto PA, Simeone E, Sileni VC et al. Clinical experience with ipilimumab $3 \mathrm{mg} / \mathrm{kg}$ : real-world efficacy and safety data from an expanded access programme cohort. J. Transl. Med. 12, 116 (2014).

40 Berrocal A, Arance A, Lopez Martin JA et al. Ipilimumab for advanced melanoma: experience from the Spanish Expanded Access Program. Melanoma Res. 24(6), 577-583 (2014).

41 Chiarion Sileni V, Pigozzo J, Ascierto PA et al. Efficacy and safety of ipilimumab in elderly patients with pretreated advanced melanoma treated at Italian centres through the expanded access programme. J. Exp. Clin. Cancer Res. 33, 30 (2014).

42 Snyder A, Makarov V, Merghoub T et al. Genetic basis for clinical response to CTLA-4 blockade in melanoma. N. Engl. J. Med. 371(23), 2189-2199 (2014).

43 Lutzky J, Wolchok J, Hamid O et al. Association between immune-related adverse events (irAEs) and disease control or overall survival in patients (pts) with advanced melanoma treated with $10 \mathrm{mg} / \mathrm{kg}$ ipilimumab in three Phase II clinical trials. 2009 American Society of Clinical Oncology (ASCO) meeting. J. Clin. Oncol. 27(15s), Abstract 9034 (2009).

44 Adaniel C, Rendleman J, Polsky D et al. Germline genetic determinants of immunotherapy response in metastatic melanoma. Presented at: ASCO Annual Meeting. Chicago, IL, USA, 30 May-3 June 2014 (Abstract 3004).

45 Di Giacomo AM, Calabro L, Danielli R et al. Long-term survival and immunological parameters in metastatic melanoma patients who responded to ipilimumab $10 \mathrm{mg} / \mathrm{kg}$ within an expanded access programme. Cancer Immunol. Immunother. 62(6), 1021-1028 (2013).

46 Yuan J, Adamow M, Ginsberg BA et al. Integrated NY-ESO-1 antibody and CD8+ T-cell responses correlate with clinical benefit in advanced melanoma patients treated with ipilimumab. Proc. Natl Acad. Sci. USA 108(40), 16723-16728 (2011).

47 Yuan J, Zhou J, Dong Z et al. Pretreatment serum VEGF is associated with clinical 
response and overall survival in advanced melanoma patients treated with ipilimumab. Cancer Immunol. Res. 2(2), 127-132 (2014). Meyer C, Cagnon L, Costa-Nunes CM et al. Frequencies of circulating MDSC correlate with clinical outcome of melanoma patients treated with ipilimumab. Cancer Immunol. Immunother. 63(3), 247-257 (2014).

49 Weber JS, Kahler KC, Hauschild A. Management of immune-related adverse events and kinetics of response with ipilimumab. J. Clin. Oncol. 30(21), 2691-2697 (2012).

50 Beck KE, Blansfield JA, Tran KQ et al. Enterocolitis in patients with cancer after antibody blockade of cytotoxic T-lymphocyteassociated antigen 4. J. Clin. Oncol. 24(15), 2283-2289 (2006).

51 Minor DR, Chin K, Kashani-Sabet M. Infliximab in the treatment of anti-CTLA4 antibody (ipilimumab) induced immunerelated colitis. Cancer Biother. Radiopharm. 24(3), 321-325 (2009).

52 Chmiel KD, Suan D, Liddle C et al. Resolution of severe ipilimumab-induced hepatitis after antithymocyte globulin therapy. J. Clin. Oncol. 29(9), e237-e240 (2011).

53 Ribas A, Hodi FS, Callahan M, Konto C, Wolchok J. Hepatotoxicity with combination of vemurafenib and ipilimumab. N. Engl. J. Med. 368(14), 1365-1366 (2013).

54 Puzanov I, Callahan MK, Linette GP et al. Phase I study of the BRAF inhibitor dabrafenib (D) with or without the MEK inhibitor trametinib $(\mathrm{T})$ in combination with ipilimumab (Ipi) for V600E/K mutationpositive unresectable or metastatic melanoma (MM). ASCO Annual Meeting. Chicago, IL, USA, 30 May-3 June 2014 (Abstract 2511).

55 Ascierto PA, Simeone E, Giannarelli D, Grimaldi AM, Romano A, Mozzillo N. Sequencing of BRAF inhibitors and ipilimumab in patients with metastatic melanoma: a possible algorithm for clinical use. J. Transl. Med. 10, 107 (2012).

56 Ackerman A, Klein O, Mcdermott DF et al. Outcomes of patients with metastatic melanoma treated with immunotherapy prior to or after BRAF inhibitors. Cancer 120(11), 1695-1701 (2014).

57 Schreuer MS, Chevolet IL, Jansen YJ et al. Objective responses can be obtained by CTLA-4 inhibition in metastatic melanoma after BRAF inhibitor failure. Melanoma Res. 25(1), 68-74 (2014).

58 Wilmott JS, Long GV, Howle JR et al. Selective BRAF inhibitors induce marked
T-cell infiltration into human metastatic melanoma. Clin. Cancer Res. 18(5), 1386-1394 (2012).

59 Frederick DT, Piris A, Cogdill AP et al. BRAF inhibition is associated with enhanced melanoma antigen expression and a more favorable tumor microenvironment in patients with metastatic melanoma. Clin. Cancer Res. 19(5), 1225-1231 (2013).

60 D’Angelo S, Dickson M, Kelly N, Yaqubie A, Chapman P. Switching therapy pre-emptively from vemurafenib (VEM) to ipilimumab (IPI) in patients (pts) with $B R A F$-mutated melanoma (MEL). Society for Melanoma Research 10th International Congress 2013. Philadelphia, PA, USA, 17-20 November 2013.

61 Chan MM, Haydu LE, Menzies AM et al. The nature and management of metastatic melanoma after progression on BRAF inhibitors: effects of extended BRAF inhibition. Cancer 120(20), 3142-3153 (2014).

62 Carlino MS, Gowrishankar K, Saunders CA et al. Antiproliferative effects of continued mitogen-activated protein kinase pathway inhibition following acquired resistance to $\mathrm{BRAF}$ and/or MEK inhibition in melanoma. Mol. Cancer Ther. 12(7), 1332-1342 (2013).

63 Zou W, Chen L. Inhibitory B7-family molecules in the tumour microenvironment. Nat. Rev. Immunol. 8(6), 467-477 (2008).

64 Hamid O, Robert C, Daud A et al. Safety and tumor responses with lambrolizumab (anti-PD-1) in melanoma. N. Engl. J. Med. 369(2), 134-144 (2013).

- This paper reports on the impressive response rates seen with the PD-1 antibody pembrolizumab in a large Phase I trial.

65 Ribas A, Hodi FS, Kefford R et al. Efficacy and safety of the anti-PD-1 monoclonal antibody pembrolizumab (MK-3475) in 411 patients (pts) with melanoma (MEL). Presented at: ASCO Annual Meeting. Chicago, IL, USA, 30 May-3 June 2014 (Abstract LBA9000).

66 Robert C, Ribas A, Wolchok JD et al. Anti-programmed-death-receptor-1 treatment with pembrolizumab in ipilimumabrefractory advanced melanoma: a randomised dose-comparison cohort of a Phase I trial. Lancet 384(9948), 1109-1117 (2014).

67 Ribas A, Puzanov I, Dummer R et al. A randomized controlled comparison of pembrolizumab and chemotherapy in patients with ipilimumab-refractory (IPI-R) melanoma (MEL). (Late breaking abstract) Presented at: Society for Melanoma Research
2014 International Congress. Zurich, Switzerland, 13-16 November 2014.

68 Topalian SL, Sznol M, Mcdermott DF et al. Survival, durable tumor remission, and long-term safety in patients with advanced melanoma receiving nivolumab. J. Clin. Oncol. 32(10), 1020-1030 (2014).

69 Topalian SL, Hodi FS, Brahmer JR et al. Safety, activity, and immune correlates of anti-PD-1 antibody in cancer. $N$. Engl. J. Med. 366(26), 2443-2454 (2012).

70 Weber JS, Minor DR, D'Angelo S et al. A Phase III randomized, open-label study of nivolumab (anti-PD-1; BMS-936558, ONO-4538) versus investigator's choice chemotherapy (ICC) in patients with advanced melanoma after prior anti-CTLA4 therapy. Ann. Oncol. 25(5), 1-41 (2014).

71 Robert C, Long GV, Brady B et al. Nivolumab in previously untreated melanoma without BRAF mutation. N. Engl J. Med. 372(4), 320-330 (2014).

-. This paper reports on a Phase III trial (in BRAF wild-type patients) clearly demonstrating the superiority of the PD-1 inhibitor nivolumab over chemotherapy in the first-line setting.

72 Atkins MB, Kudchadkar RR, Sznol M et al. Phase II, multicenter, safety and efficacy study of pidilizumab in patients with metastatic melanoma (abstract 9001). Presented at: ASCO Annual Meeting 2014. Chicago, IL, USA, 30 May-3 June 2014.

73 Brahmer JR, Tykodi SS, Chow LQ et al. Safety and activity of anti-PD-L1 antibody in patients with advanced cancer. $N$. Engl. J. Med. 366(26), 2455-2465 (2012).

74 Hamid O, Sosman JA, Lawrence DP et al. Clinical activity, safety, and biomarkers of MPDL3280A, an engineered PD-L1 antibody in patients with locally advanced or metastatic melanoma (abstract \#9010). Presented at: ASCO Annual Meeting 2013. Chicago, USA, 31 May-4 June 2013.

75 Chan MM, Kefford RF, Carlino M, Clements A, Manolios N. Arthritis and tenosynovitis associated with the anti-PD1 antibody pembrolizumab in metastatic melanoma. J. Immunother. 38(1), 37-39 (2015).

76 Wolchok JD, Kluger H, Callahan MK et al Nivolumab plus ipilimumab in advanced melanoma. N. Engl. J. Med. 369(2), 122-133 (2013).

- This paper reports the impressive responses seen in a Phase I trial of the combination of nivolumab and ipilimumab, and highlights the increased toxicity seen with the combination. 
77 Sznol M, Kluger HM, Callahan MK et al. Survival, response duration, and activity by BRAF mutation (MT) status of nivolumab (NIVO, anti-PD-1, BMS-936558, ONO4538) and ipilimumab (IPI) concurrent therapy in advanced melanoma (MEL) (abstract LBA9003). Presented at: American Society of Clinical Oncology (ASCO) Annual Meeting 2014. Chicago, IL, USA, 30 May-3 June 2014.

78 Taube JM, Klein A, Brahmer JR et al. Association of PD-1, PD-1 ligands, and other features of the tumor immune microenvironment with response to anti-PD-1 therapy. Clin. Cancer Res. 20(19), 5064-5074 (2014).

79 Kefford R, Ribas A, Hamid O et al. Clinical efficacy and correlation with tumor PD-L1 expression in patients (pts) with melanoma (MEL) treated with the anti-PD-1 monoclonal antibody MK-3475 (abstract 3005). Presented at: American Society of Clinical Oncology (ASCO) Annual Meeting 2014. Chicago, IL, USA, 30 May-3 June 2014.

80 Tumeh PC, Harview CL, Yearley JH et al. PD-1 blockade induces responses by inhibiting adaptive immune resistance. Nature 515(7528), 568-571 (2014).
81 Eggermont AM, Chiarion-Sileni V, Grob JJ et al. Ipilimumab versus placebo after complete resection of stage III melanoma: initial efficacy and safety results from the EORTC18071 Phase III trial (abstract LBA9008). Presented at: American Society of Clinical Oncology (ASCO) Annual Meeting 2014. Chicago, IL, USA, 30 May-3 June 2014.

82 Hodi FS, Lee S, Mcdermott DF et al. Ipilimumab plus sargramostim vs ipilimumab alone for treatment of metastatic melanoma: a randomized clinical trial. JAMA 312(17), 1744-1753 (2014).

83 Puzanov I, Milhem MM, Andtbacka RHI et al. Primary analysis of a Phase Ib multicenter trial to evaluate safety and efficacy of talimogene laherparepvec (T-VEC) and ipilimumab (ipi) in previously untreated, unresected stage IIIB-IV melanoma (abstract 9029). Presented at: American Society of Clinical Oncology (ASCO) Annual Meeting 2014. Chicago, IL, USA, 30 May-3 June 2014.

84 Postow MA, Callahan MK, Barker CA et al. Immunologic correlates of the abscopal effect in a patient with melanoma. N. Engl. J. Med. 366(10), 925-931 (2012).

85 Grimaldi AM, Simeone E, Giannarelli D et al. Abscopal effects of radiotherapy on advanced melanoma patients who progressed after ipilimumab immunotherapy. Oncoimmunology 3, e28780 (2014).

86 Barker CA, Postow MA, Khan SA et al. Concurrent radiotherapy and ipilimumab immunotherapy for patients with melanoma. Cancer Immunol. Res. 1(2), 92-98 (2013).

87 Sznol M, Hodi FS, Margolin K et al. Phase I study of BMS-663513, a fully human anti-CD137 agonist monoclonal antibody, in patients with advanced cancer. Presented at: 44th Annual Meeting of the American Society of Clinical Oncology 2008. Chicago, IL, USA, 30 May-3 June 2008 (Abstract 3007).

88 Fan X, Quezada SA, Sepulveda MA, Sharma $P$, Allison JP. Engagement of the ICOS pathway markedly enhances efficacy of CTLA- 4 blockade in cancer immunotherapy. J. Exp. Med. 211(4), 715-725 (2014).

89 Derre L, Rivals JP, Jandus C et al. BTLA mediates inhibition of human tumor-specific $\mathrm{CD} 8+\mathrm{T}$ cells that can be partially reversed by vaccination. J. Clin. Invest. 120(1), 157-167 (2010).

90 Chen L, Flies DB. Molecular mechanisms of $T$ cell co-stimulation and co-inhibition. Nat. Rev. Immunol. 13(4), 227-242 (2013). 